\title{
SAÚDE BUCAL: CONHECIMENTO E IMPORTÂNCIA PARA A GESTANTE
}

\author{
ORAL HEALTH: KNOWLEDGE AND IMPORTANCE FOR PREGNANT WOMEN
}

\author{
Francisco Cezanildo Silva Benedito ${ }^{a^{*}}$, Davide Carlos Joaquim ${ }^{b^{*}}$, Ana Gesselena da Silva Farias ${ }^{c^{*}}$, \\ Edmara Chaves Costa ${ }^{\mathrm{d}^{*}}$, Erika Helena Salles de Brito ${ }^{\mathrm{e}^{*}}$, Ana Caroline Rocha de Melo Leite ${ }^{\mathrm{f}^{*}}$ \\ acezanildo.silvab@outlook.com, ${ }^{\mathrm{a}}$ davidejoaquim@hotmail.com, ${ }^{\mathrm{c}}$ gessefarias@hotmail.com, \\ dedmaracosta@unilab.edu.br, ${ }^{\mathrm{d}}$ erika@unilab.edu.br, ${ }^{\dagger}$ acarolmelo@unilab.edu.br \\ *Universidade da Integração Internacional da Lusofonia Afro-Brasileira - Redenção (CE), Brasil
}

Data de recebimento do artigo: 02/02/2017

Data de aceite do artigo: 25/04/2017

\section{RESUMO}

Introduçáo: Durante a gestação, alteraçôes fisiológicas, hormonais, psicológicas e comportamentais podem repercutir sobre a saúde bucal da mãe, interferindo na saúde do bebê. Objetivo: Caracterizar o conhecimento de gestantes sobre diferentes aspectos da saúde bucal, acompanhadas pela Enfermagem. Materiais e métodos: Estudo descritivo, prospectivo e quantitativo, realizado com 32 gestantes atendidas em uma Unidade Básica de Saúde da Família de um município cearense. Os dados foram analisados, interpretados e descritos. Resultados: A populaçáo foi composta, em sua maioria, por gestantes maiores de idade, casadas, com escolaridade variando entre ensino fundamental incompleto e superior completo, e renda de até três salários mínimos. Das participantes, 68,7\% desconheciam as doenças que atingem a cavidade oral, e todas utilizavam escova e creme dental. Quanto aos meios preventivos, $75 \%$ das gestantes indicaram escovação e uso do fio dental e 43,7\% mencionaram busca pelo cirurgiáo-dentista. Do total, 53,1\% apresentavam sangramento gengival na escovação e 59,4\% já tinham participado de açóes de Educação em Saúde, realizadas especialmente pelo odontólogo. No tocante à relação gravidez, saúde bucal materna e saúde do bebê, a maioria das gestantes era consciente. Práticas corretas de higiene oral do bebê foram apontadas por 90\% das pesquisadas, $68,7 \%$ mencionaram a erupção do primeiro dente como momento ideal de levar o bebê à primeira consulta odontológica, e 87,5\% apresentavam dieta cariogênica. Conclusóes: A maioria das gestantes desconhecia as doenças que atingem a cavidade oral, mas sabiam como evitá-las. Elas eram conscientes da relação entre gravidez, saúde oral materna e saúde do bebê.

Palavras-chave: Saúde bucal; gestação; enfermagem em saúde comunitária.

\section{ABSTRACT}

Introduction: During gestation, physiological, hormonal, psychological and behavioral changes may affect the mother's oral health, interfering with the baby's health. Objective: To characterize the knowledge of pregnant women, about different aspects of oral health, accompanied by Nursing. Materials and methods: Descriptive, prospective and quantitative study with 32 pregnant women attended at a Basic Family Health Unity in a city Ceará. The data were analyzed, interpreted and described. Results: The majority of the population consisted of pregnant of legal age, married, with schooling varying between incomplete elementary and higher education and income of up to three minimum wages. Of the participants, $68.7 \%$ didn't know the diseases that reach the oral cavity and all used brush and toothpaste. Regarding preventive measures, $75 \%$ of pregnant indicated brushing and flossing, and $43.7 \%$ mentioned a search by the dentist. Of the total, $53.1 \%$ had gingival bleeding on toothbrushing and 59.4\% had already participated in Health Education actions, performed especially by the dentist. Regarding the relationship among pregnancy, maternal oral health and baby health, the majority of pregnant was aware. Correct baby oral hygiene practices were pointed out by $90 \%$ of those surveyed, $68.7 \%$ mentioned the eruption of the first tooth as an ideal moment to take the baby to the first dental appointment, and $87.5 \%$ presented a cariogenic diet. Conclusions: Most pregnant were unaware of diseases that reach the oral cavity, but they knew how to avoid them. They were aware of the relationship among pregnancy, maternal oral health and baby health.

Keywords: Oral health; gestation; nursing in community health. 


\section{Introdução}

Ao longo da história, o conceito de saúde evoluiu, acompanhando e refletindo o cenário social, político, econômico e cultural de cada época. Nesse contexto, a Organização Mundial da Saúde (OMS), em sua carta de princípios, de 7 de abril de 1948, definiu a saúde como completo estado de bem-estar físico, mental e social, não se restringindo à ausência de doença ${ }^{1}$. Apesar das críticas, esse conceito despertou para o fato de que a saúde resulta da ação de fatores biológicos, econômicos, sociais, políticos e culturais.

Nessa nova forma de pensar o processo saúde-doença, reconhece-se a saúde bucal como parte integrante e essencial da saúde geral. De fato, manifestaçôes bucais podem ocorrer como sinais e sintomas iniciais de doenças ou alterações sistêmicas, funcionando como meio de alerta. Em contrapartida, essas condiçóes podem desenvolver-se pela ação de microrganismos oriundos da cavidade oral ${ }^{2}$.

No âmbito dessa correlação saúde geral-saúde bucal, a gestação figura como exemplo claro ao se observar que, durante esse período, a cavidade oral da mulher passa por alteraçóes fisiológicas capazes de contribuir para o desenvolvimento de doenças bucais, como cárie e doenças periodontais (gengivite e periodontite) ${ }^{3,4}$. Essas patologias, embora associadas à elevada perda dentária, podem ser facilmente prevenidas por práticas de higiene oral adequadas 5 .

No tocante ao aumento da suscetibilidade ao processo carioso na gravidez, alteraçóes do hábito alimentar da futura mãe, como aumento do consumo de açúcar, e/ou descuido com sua higiene oral, podem justificar tal vulnerabilidade. Contribuem ainda para esse aumento o surgimento de alteraçôes salivares, como a mudança da composição e diminuição da secreção salivar, e hormonais, como o aumento dos níveis de progesterona e estrógeno ${ }^{6}$.

Concomitantemente, a vulnerabilidade às doenças periodontais acontece pela alteração da vascularização gengival, como consequência dos níveis elevados de progesterona e estrógeno, promovendo hiperemia, edema local e gengivite ${ }^{7,8}$. O agravamento dessas enfermidades pode associar-se à depressão do sistema imune e à alteração da composição da microbiota supra e subgengival'.

Em contrapartida, os patógenos periodontais podem se disseminar pelo organismo da futura mãe, tornando-a vulnerável ao desenvolvimento de condiçóes diretamente relacionadas ao seu estado, como pré-eclâmpsia, parto prematuro e baixo peso do bebê ao nascer ${ }^{8,10}$. Essa propagação pode provocar também desordens sistêmicas, independente do estado gestacional, como, por exemplo, artrite, doenças cardiovasculares, diabetes, aterosclerose e outras ${ }^{9}$. Assim, é nítido que a assistência integral à gestante deve ser prioridade para os profissionais de saúde, visando ao bom desenvolvimento gestacional e a geração de filhos saudáveis.

Nesse contexto, ressalta-se a importância do profissional de Enfermagem, por seu papel fundamental no acompanhamento da gestante em seu período pré-natal, bem como sua facilidade para estabelecer vínculos e seu compromisso com o cuidado cotidiano com a higiene bucal ${ }^{11}$.

Dessa forma, a Enfermagem deve agir em conjunto com Odontologia, por meio da troca de saberes e informaçóes entre a gestante e o enfermeiro, buscando a promoção do autocuidado ${ }^{12}$.

Assim, este estudo teve como objetivo caracterizar o conhecimento de gestantes sobre diferentes aspectos da saúde bucal, acompanhadas pela Enfermagem, em uma Unidade Básica de Saúde de um município cearense.

\section{Metodologia}

Trata-se de um estudo descritivo, prospectivo e de abordagem quantitativa. A amostra foi composta por 32 gestantes atendidas no Centro de Saúde de Acarape, localizado no município de Acarape (CE). A coleta de dados foi realizada por acadêmico de Enfermagem da Universidade da Integração Internacional da Lusofonia Afro-Brasileira (Unilab), no período de setembro de 2014 a agosto de 2015.

Adotou-se como critério de inclusão a gestante estar frequentando regularmente a consulta pré-natal e, como critérios de exclusão, a paciente possuir alguma limitação cognitiva que inviabilizasse a coleta, ou ser menor de idade e estar desacompanhada de um responsável no momento da consulta.

No período em que o estudo foi conduzido, das 47 gestantes atendidas na unidade básica de saúde, 32 aceitaram participar do estudo, 8 se recusaram, 4 tinham fixado residência em outro município e 3 não foram contatadas, por náo comparecerem regularmente às consultas pré-natais.

A coleta de dados ocorreu por meio da aplicaçáo de um questionário estruturado, abordando os seguintes aspectos: perfil epidemiológico; conhecimento das doenças bucais e sua relação com a gravidez; meios utilizados para a higiene oral; conhecimento de meios preventivos; presença de sangramento gengival no ato da escovação dentária; participação prévia em açóes de Educação em Saúde; conscientização quanto à relação gestação, saúde bucal materna e saúde do bebê; conhecimento quanto aos cuidados em saúde bucal do bebê; dieta.

Os dados foram devidamente tabulados, analisados pelo programa Epi Info versão 7, interpretados e descritos. 
O projeto de pesquisa foi aprovado pelo Comitê de Ética em Pesquisa da Unilab, conforme CAAE 26146213.6.0000.5576 e parecer no 566.465. O desenvolvimento do estudo seguiu os princípios da Resolução no 466/2012 do Conselho Nacional de Saúde (CNS), que estabelece os preceitos éticos das pesquisas envolvendo seres humanos.

\section{Resultados e discussão}

De acordo com as informaçóes coletadas, o perfil epidemiológico mostrou que $9,4 \%$ das gestantes eram menores de idade. A média de idade das demais participantes variou de 25 a 33 anos, com 12,5\% delas apresentando 31 anos. Em relação à naturalidade e estado civil, $65,6 \%$ das grávidas eram naturais do município de Redenção - CE e 59,4\% eram casadas ou estavam em uniáo estável. O grau de escolaridade incluiu pacientes com Ensino Fundamental incompleto $(18,7 \%)$ e completo (15,6\%), Ensino Médio incompleto (12,5\%) e completo (31,3\%), Ensino Superior incompleto $(6,3 \%)$ e completo $(15,6 \%)$. Os dados revelaram ainda que $43,7 \%$ das pesquisadas tinham renda familiar entre $1 \mathrm{e}$ 3 salários mínimos; 34,4\% menos de um salário mínimo e $21,9 \%$ não tinham rendimento algum.

O perfil socioeconômico apresentado assemelhou-se ao de gestantes atendidas no serviço público de saúde de São Luís, estado do Maranhão, exceto o grau de escolaridade. Quanto a este, os autores relataram que apenas $10,7 \%$ das participantes usuárias desse tipo de serviço tinham mais de 10 anos de estudo, quantitativo inferior ao aqui observado ${ }^{13}$.

O estudo mostrou que $68,7 \%$ das gestantes desconheciam as doenças que atingem a cavidade oral. Tal dado é particularmente importante se considerada a relaçáo entre saúde oral e gravidez. De fato, as doenças que atingem a cavidade bucal - como a periodontite, entendida como uma doença inflamatória que acomete o tecido de sustentação dos dentes -podem provocar efeitos indesejados sobre a gestação, tais como pré-eclâmpsia, parto prematuro e baixo peso do bebê ao nascer ${ }^{14}$.

A relevância desse resultado também reside no fato de que há maior prevalência de problemas bucais em gestantes, quando comparado à população em geral ${ }^{15}$.

Diante das possíveis repercussóes dos problemas bucais sobre a saúde da futura mãe, e maior suscetibilidade desta perante as patologias orais, surge a necessidade de divulgar a esse público informaçōes referentes à saúde bucal, no sentido de evitar consequências negativas durante e após o período gestacional.

Das participantes que desconheciam as enfermidades bucais, $65,2 \%$ não tinham rendimento, ou esse era inferior a um salário mínimo. Considerando que o estado de vulnerabilidade socioeconômica torna indivíduos mais suscetíveis à aquisição de patologias, haja vista que náo dispóem de recursos para promoverem seu bem-estar ${ }^{16}$, a falta de conhecimento e as condiçôes econômicas da maioria das gestantes desse estudo aumentam os riscos de desenvolvimento de problemas bucais e suas consequências.

Além do conhecimento e condiçóes econômicas, o tipo de comportamento adotado pela futura mãe pode influenciar sua saúde bucal e a do seu bebê. Corroborando com essa suposição, Gonik et al. ${ }^{17}$ avaliaram o conhecimento e comportamento de gestantes em relação à saúde oral perinatal e concluíram que muitas praticaram comportamentos de risco capazes de comprometer a saúde bucal e ampliar as chances de seus filhos desenvolverem cárie infantil.

Quando questionadas sobre o que utilizavam para realizar sua higiene oral, todas referiram escova e creme dental. No entanto, o fio dental foi mencionado por apenas $34,4 \%$ das pesquisadas, das quais $54,5 \%$ apresentavam renda entre um e três salários mínimos. Tal achado reforça as consideraçóes de Barros e Nicolau ${ }^{16}$ ao relacionarem os fatores socioeconômicos, como baixa renda, escolaridade e estado civil à saúde.

A baixa referência ao uso do fio dental não foi surpreendente, pois a literatura aponta reduzida prática pela população, incluindo gestantes ${ }^{13,15,18}$. É possível que esse fenômeno ocorra pelo fio dental ser considerado meio auxiliar na remoção da placa bacteriana ${ }^{19}$, bem como a falta de introdução desse hábito à criança pelos pais ou responsáveis ou sensação de limpeza após a escovação dentária ${ }^{20}$.

Neste estudo, 59,4\% das participantes afirmaram conhecer alguma forma de evitar doenças bucais. A importância desse resultado se dá pelo fato de que, ao conhecerem formas de prevenção, as futuras mães podem aprimorar sua saúde oral no período pré-natal, repercutindo positivamente sobre sua saúde geral e a do bebê. Ainda, como consequência, pode-se minimizar o risco potencial de transmissão de bactérias da microbiota oral da mãe ao filho, e contribuir para a instituição de hábitos bucais saudáveis ${ }^{21}$.

Escovar os dentes e utilizar fio dental foi apontado por $75 \%$ das pacientes como forma de prevenção das patologias orais, percentual semelhante ao obtido por Asa'ad et al. ${ }^{22}$. A referência à escovação e utilização de fio dental condiz com a literatura, ao mencionar que o uso regular desses meios de higienização tem capacidade de eliminar bactérias e substâncias cariogênicas da superfície dentária, dificultando a formação do biofilme e, consequentemente, o desenvolvimento da cárie ${ }^{23}$.

Embora a procura pelo cirurgião-dentista tenha sido referida por $43,7 \%$ das participantes como meio 
preventivo, estudos mostram que, mesmo diante da necessidade de consulta odontológica, a gestante não busca atendimento, ou o faz apenas quando sente dor ${ }^{13,24}$. É possível que essa escassa procura pelo odontólogo decorra do medo que a maioria das pessoas tem desse profissional, e/ou da crença de que gestantes não podem ser submetidas a tratamento odontológico, bem como falta de tempo com as demandas domésticas e cuidado com outros filhos ${ }^{15,25,26}$.

Ao responderem sobre sangramento gengival, $53,1 \%$ das pesquisadas afirmaram tê-lo durante a escovação. Esse dado reforça a afirmação de que a gestação acarreta mudanças no estado gengival das pacientes, refletidas na forma de hiperemia, edema e sangramento. De fato, o aumento da taxa do metabolismo de estrógeno e síntese de prostaglandinas pela gengiva contribui para as alterações gengivais observadas durante a gravidez.

Corroborando o que foi anteriormente mencionado, um estudo com 415 mulheres grávidas evidenciou presença de sangramento gengival em $56,3 \%$ das participantes, na faixa etária de 35-44 anos ${ }^{3}$.

Vale ressaltar que a ocorrência de gengivite gravídica varia de $30 \%$ a $86 \%{ }^{27,28}$, e, caso não tratada, pode evoluir para periodontite, doença que tem sido associada a efeitos adversos da gravidez ${ }^{29,30}$.

Apesar dessas evidências, é possível que a gestante não tenha consciência de que a maior predisposiçáo ao sangramento gengival seja consequência de seu estado atual, conforme observado por Lopes et al. ${ }^{13}$.

Os dados revelaram que $59,4 \%$ das pacientes já tinham participado de ações de Educação em Saúde. Quando indagadas sobre o profissional responsável por tal ação, 78,9\% indicaram o cirurgião-dentista. Nenhuma das participantes apontou o(a) enfermeiro(a) como agente educador, sugerindo deficiência na atuação desse profissional em relação à saúde bucal.

Esse resultado reflete claramente o que aponta um estudo sobre a percepção do enfermeiro sobre a saúde bucal na gestação, que afirma que esse profissional apresenta incerteza e inexperiência quanto à prática de atenção nesse tipo de saúde ${ }^{31}$.

Corroborando essa suposição, uma pesquisa recente apontou conhecimento limitado de enfermeiras sobre saúde bucal e sua relação com a gravidez ${ }^{32}$, embora fossem conscientes quanto aos cuidados necessários à futura mãe.

Ao serem questionadas sobre a influência da gestação na saúde bucal, $68,7 \%$ das mulheres pesquisadas apontaram estar cientes. Em relação ao fato de sua saúde bucal afetar a saúde do seu filho, $62,5 \%$ das gestantes relataram ser conscientes. Esses resultados contrastam com dados da literatura, nos quais um reduzido número de gestantes era consciente da relação saúde bucal, gravidez e saúde do bebê $\hat{1}^{13,15,17}$.

Quanto ao conhecimento sobre a higienização da cavidade oral do bebê, $90 \%$ das participantes apontaram utilização de gaze ou fralda limpa e água filtrada. Esse resultado surpreendente se opóe ao de Wapniarska, Bula e Hilt ${ }^{33}$, que revelaram uma deficiência no conhecimento de futuros pais sobre a prevençáo e higiene oral de crianças, indicando, portanto, necessidade de treinamentos especiais. Para os pesquisadores, esses devem ser feitos não apenas pelo odontólogo, mas também por pediatras, enfermeiros e médicos que realizam as consultas de pré-natal.

A discrepância encontrada entre esses estudos pode relacionar-se ao fato de que as gestantes desta pesquisa já tinham recebido orientaçóes prévias, realizadas principalmente pelo cirurgião-dentista. No entanto, o limitado número de participantes pode ter contribuído para esse resultado.

Ao responderem sobre quando deveriam levar o bebê ao consultório odontológico pela primeira vez, $68,7 \%$ indicaram o momento em que ocorre a erupção do primeiro dente. Esse dado reforça a necessidade de uma avaliação da saúde oral do bebê o mais rápido possível, tendo em vista, se necessária, uma intervenção antecipada e assertiva. Essa busca antecipada ainda pode contribuir para a realização de açóes educativas com os pais, visando a prevenção de doenças e promoção da saúde ${ }^{34}$.

Das participantes, $87,5 \%$ apresentaram consumo excessivo de alimentos ricos em carboidratos, especialmente arroz, milho, macarrão, pão, batata-inglesa e macaxeira. Quanto ao consumo de outros alimentos, os resultados mostraram que $31,2 \%$ e $18,7 \%$ das gestantes ingerem verduras e grãos, respectivamente.

Com base nessas evidências, pode-se perceber que elevado número de gestantes consome alimentos ricos em carboidratos, em detrimento do consumo de alimentos não cariogênicos, aumentando o risco do desenvolvimento de processos cariosos ${ }^{6}$. Além do desenvolvimento de processos cariosos, o consumo excessivo de alimentos ricos em carboidratos pode contribuir significativamente para o desenvolvimento da obesidade.

Nesse âmbito, é importante ainda mencionar que a nutrição adequada é uma condição crucial para o desenvolvimento saudável da gestação, pois, nesse período, a mulher vivencia alterações metabólicas, hormonais e psicológicas que podem ser influenciadas pela alimentação. Dessa forma, uma dieta equilibrada contribui sobremaneira para o crescimento adequado do feto e para a preservação da saúde materna, em especial a saúde oral ${ }^{35}$. 


\section{Conclusões}

O estudo revelou que grande parte das gestantes desconheciam as doenças que atingem a cavidade oral, embora muitas conhecessem como evitá-las. Elas utilizavam escova e creme dental para higienização da cavidade bucal, e apresentavam sangramento gengival no momento da escovação. Já tinham participado de açôes de Educação em Saúde, conduzidas especialmente pelo cirurgião-dentista.

As participantes eram conscientes quanto à influência da gestação sobre a saúde bucal e desta sobre a saúde do seu filho. Conheciam os métodos utilizados na higienização bucal do bebê, porém desconheciam a importância de uma dieta adequada para o crescimento do feto e preservação da saúde materna.

Ressalta-se que a saúde bucal da gestante é de extrema importância para a formação de crianças, adolescentes e adultos saudáveis. Para tanto, faz-se necessário o conhecimento sobre a temática pela Enfermagem, profissão fortemente atuante nas consultas pré-natal, que, agindo conjuntamente com o odontólogo, pode promover uma saúde bucal de qualidade a esse público.

\section{Agradecimentos}

Agradecemos às gestantes que dedicaram parte do seu tempo para o preenchimento do questionário e participação seguinte nas ações de Educação em Saúde, realizadas pelo acadêmico de Enfermagem. Ficamos gratos ainda pela imensa colaboração das enfermeiras do município de Acarape: dra. Aline Maria Diogenes Pessoa e dra. Brena Luiza Gomes de Castro, e aos demais profissionais do Centro de Saúde de Acarape. Agradecemos ainda ao Programa de Educação pelo Trabalho para a Saúde/Vigilância em Saúde (PET Saúde/VS) da Unilab, pela incontestável oportunidade de crescimento acadêmico, profissional e pessoal.

\section{Referências}

1. World Health Organization. Preamble to the Constitution of the World Health Organization as adopted by the International Health Conference, New York, 19-22, June 1946, and entered into force on 7 April 1948.

2. Wadhawan R, Solanki G, Sabir S, Palekar S, Pareekh A. Oral manifestations of systemic diseases: a review. Journal of Science. 2014;4(4):233-41.

3. Onigbinde OO, Sorunke ME, Braimoh MO, Adeniyi AO. Periodontal status and some variables among pregnant women in a Nigeria tertiary institution. Ann Med Saúde Sci Res. 2014;4(6):852-7.
4. Wu M, Chen SW, Jiang SY. Relationship between gingival inflammation and pregnancy. Mediators Inflamm. 2015;2015:1-11.

5. Moyer VA. Prevention of dental caries in children from birth through age 5 years: us preventive services task force recommendation statement. Pediatrics. 2014;133(6):1-10.

6. Oliveira LB, Moreira RS, Reis SCGB, Freire MCM. Cárie dentária em escolares de 12 anos: análise multinível dos fatores individuais e do ambiente escolar em Goiânia. Rev Bras Epidemiol. 2015;18(3):642-54.

7. Ramos ESM, Martins NR, Kroumpouzos G. Oral and vulvovaginal changes in pregnancy. Clin Dermatol. 2016;34(3):353-8.

8. Umoh AO, Savage KO, Ojehanon PI. Association between maternal gingivitis, low birth weight and preterm delivery. JMBR.2013;12(1):65-75.

9. Albornoz AC, Figuero E, Herrera D, Martínez AB. Gingival changes during pregnancy: II. Influence of hormonal variations on the subgingival biofilm. J Clin Periodontol. 2010;37(3):230-40.

10. Zi MYH, Longo PL, Silva BB, Mayer MPA. Mechanisms involved in the association between periodontitis and complications in pregnancy. Front Public Health. 2014;2:290.

11. Araújo MVM, Vieira MA, Bonan PRF, Costa SM. Atuação dos profissionais de enfermagem nos cuidados com a higiene bucal de idosos institucionalizados em montes claros MG. Rev APS. 2010;13(1):10-7.

12. Hartnett E, Haber J, Miller KB, Bella A, Vasilyeva A, Kessler JL. Oral Health in Pregnancy. J Obstet Gynecol Neonatal Nurs. 2016;45:565-73.

13. Lopes FF, Ribeiro TV, Fernandes DB, Calixto NRV, Alves CMC, Pereira ALA, et al. Oral health knowledge and practices among pregnant women using health services in São Luís, Maranhão, Brazil, 2007-2008. Epidemiol Serv Saúde. 2016;25(4):819-26.

14. Eke PI, Dye BA, Wei L, Slade GD, Evans GOT, Borgnakke WS, et al. Update on Prevalence of Periodontitis in Adults in the United States: NHANES 2009 to 2012. J Periodontol. 2015;86(5):611-22.

15. George A, Johnson M, Blinkhorn A, Ajwani S, Bhole S, Yeo A, et al. The oral health status, practices and knowledge of pregnant women in south-western Sydney. Aust Dent J. 2013;58(1):26-33.

16. Barros MAR, Nicolau AIO. Fatores socioeconômicos da gestante associados ao peso do recém-nascido. Rev enferm UFPE on line. 2013;7(7):4769-74.

17. Gonik B, Wilson E, Mayberry M, Joarder BY. Pregnant Patient Knowledge and Behavior Regarding Perinatal Oral Health. Am J Perinatol. 2016;34(7):663-667.

18. Kubo FMM, Mialhe FL. Fio dental: da dificuldade ao êxito na remoção do biofilme interproximal. Arq Odontol. 2011;47(1):51-5.

19. Silva EM, Filho CE, Nepomuceno VC. Uma grande descoberta: $\mathrm{O}$ prazer que a higienização bucal correta e 
bem orientada pode proporcionar. Rev Odontológica de Araçatuba. 2003;24(2):39-42.

20. Srinivas SR, Singhal R, Kaur N. Causes for non-usage of floss among students in a dental institution in North India: A questionnaire study. PROBE. 2014;48(3):109-14.

21. Riggs E, Yelland J, Shankumar R, Kilpatrick N. We are all scared for the baby: promoting access to dental services for refugee background women during pregnancy. BMC Pregnancy Childbirth. 2016;16(12):1-11.

22. Asa'ad FA, Rahman G, Mahmoud N, Shamasi E, Khuwaileidi A. Periodontal disease awareness among pregnant women in the central and eastern regions of Saudi Arabia. J Investig Clin Dent. 2015;6(1):8-15.

23. Castilho ARF, Mialhe FL, Barbosa TS, Puppin-Rontani RM. Influence of Family environment on children's oral health: a systematic review. J Pediatr (Rio J). 2013;89(2):116-23

24. Malkawi ZA, Tubaishat RS. Knowledge, practice and utilization of dental services among pregnant women in the north of Jordan. J Contemp Dent Pract. 2014;15(3):345-51.

25. Bamanikar S, Kee LK. Knowledge, attitude and practice of oral and dental healthcare in pregnant women. Oman Med J. 2013;28(4):288-91.

26. Thomas NJ, Middleton PF, Crowther CA. Oral and dental health care practices in pregnant women in Australia: a postnatal survey. BMC Pregnancy Childbirth. 2008;8(13):1-6.

27. Rakchanok N, Amporn D, Yoshida Y, Harun-Or-Rashid M, Sakamoto J. Dental caries and gingivitis among pregnant and non-pregnant women in Chiang Mai, Thailand. Nagoya J Med Sci. 2010;72(1-2):43-50.

28. Barak S, Oettinger-Barak O, Oettinger M, Machtei EE, Peled M, Ohel G. Common oral manifestations during pregnancy: a review. Obstet Gynecol Surv. 2003;58(9):624-8.

29. Pitiphat W, Joshipura KJ, Gillman MW, Williams PL, Douglass CW, Rich-Edwards JW. Maternal periodontitis and adverse pregnancy outcomes. Community Dent Oral Epidemiol. 2008;36(1):3-11.

30. Saddki N, Bachok N, Hussain NHN, Zainudin SLA, Sosroseno W. The association between maternal periodontitis and low birth weight infants among Malay women. Community Dent Oral Epidemiol. 2008;36(4):296-304.

31. Venancio EQ, Paula EMQV, Imada SR, Reis CB. A percepção do enfermeiro da saúde da família sobre saúde bucal na gestação. Ciênc Cuid Saúde. 2011;10(4):812-9.

32. Sharif S, Saddki N, Yusoff A. Knowledge and Attitude of Medical Nurses toward Oral Health and Oral Health Care of Pregnant Women. Malays J Med Sci. 2016;23(1):63-71.

33. Wapniarska K, Buła K, Hilt A. Parent's pro-health awareness concerning oral health of their children in the light of survey research. Przegl Epidemiol. 2016;70(1):59-63.

34. American Academy of Pediatric Dentistry. Guideline on infant oral health care. Clin Pract Guidel Quick Ref Guide Clin. 2014;37(6):146-50.

35. Nada M, Dragan T, Bojana K. Nutritional analysis in women during pregnancy. Researches Review DGTH. 2015;44(2),183-93.

\section{Como citar este artigo:}

Benedito FCS, Joaquim DC, Farias AGS, Costa EC, Brito EHS, Leite ACRM. Saúde bucal: conhecimento e importância para a gestante. Rev. Aten. Saúde. 2017;15(52):43-48. 